\title{
TFIIIC determines RNA polymerase III specificity at the TATA-containing yeast U6 promoter
}

\author{
Sadia Roberts, Trenton Colbert, and Steven Hahn ${ }^{1}$ \\ Fred Hutchinson Cancer Research Center, Seattle, Washington 98104 USA
}

\begin{abstract}
The gene encoding yeast U6 snRNA that is transcribed by RNA polymerase III (Pol III) contains both a TATA box upstream of the transcription start site and a downstream binding site for the factor TFIIIC. This juxtaposition of elements typical of both Pol II- and Pol III-transcribed genes raises the question of how polymerase specificity is determined. The upstream U6 promoter containing the TATA box and transcription start site was shown previously to be transcribed by Pol III in vitro. We therefore tested whether the upstream promoter of yeast U6 encodes Pol III specificity. One model is that polymerase specificity is conferred by the homologous Pol II and Pol III transcription factors TFIIB and BRF1. However, we found no specificity in the binding of BRF1 or TFIIB to TATA-containing promoters of genes specifically transcribed by Pol III or Pol II. Yeast strains deficient for Pol II or Pol III transcription were employed to examine U6 polymerase specificity in vivo. We find that the U6 upstream promoter is Pol II-specific in vivo and is converted to Pol III specificity by TFIIIC. Thus, preferential recruitment of TFIIIB by TFIIIC probably excludes the Pol II general factors and promotes Pol III transcription, thereby determining polymerase specificity.
\end{abstract}

[Key Words: RNA polymerase specificity; TFIIIC; TATA box; U6 promoter]

Received December 21, 1994; revised version accepted February 22, 1995.

Transcription of the majority of eukaryotic genes by the nuclear RNA polymerases Pol I, Pol II, and Pol III requires three structurally distinct types of promoter, each specific for one class of polymerase. Thus, historically, recruitment of three distinct classes of transcription factors was assumed to determine polymerase specificity. In recent years, these distinctions have been blurred by descriptions of common promoter elements, as well as common or related transcription factors, shared by genes in the different polymerase classes. For instance, TATAbinding protein (TBP) is an essential component of at least three transcription factor complexes that are specific for genes transcribed by RNA Pol I, Pol II, and Pol III (for review, see in Hernandez 1993; Rigby 1993). These complexes, SLl, TFIID, and TFIIIB, respectively, consist of TBP and several TBP-associated factors (TAFs). In addition, at least two polymerase-specific factors are related: A component of TFIIIB called BRF1 (also known as TDS4 or PCF4) has homology with the Pol II factor TFIIB (Buratowski and Zhou 1992; Colbert and Hahn 1992; Lopez-De-Leon et al. 1992). Polymerase specificity is probably mediated by recruitment of specific TBP/TAF complexes by promoter elements unique to each gene class. For example, in tRNA genes, TFIIIB is recruited by intragenic promoter elements that bind transcription

\footnotetext{
${ }^{1}$ Corresponding author.
}

factor TFIIIC and that are found exclusively in Pol III genes (Geiduscheck and Tocchini-Valentini 1988; Geiduschek and Kassavetis 1992).

In contrast, however, a class of Pol III transcription unit exemplified by the genes encoding U6 snRNA from vertebrates to yeast contains promoter elements shared by Pol II genes. In vertebrates, the promoters of snRNA genes are dissimilar from most other Pol III promoters in that they reside entirely upstream of the site of transcription initiation (for review, see Hernandez 1992). The promoters of the vertebrate U1 and U2 snRNA genes comprise a distal enhancer element (DSE) and a proximal element (PSE), which specifically promote Pol II transcription. The U6 promoter contains three elements, a DSE, a PSE, and a TATA box, all of which are also found in Pol II promoters. Surprisingly, the TATA box determines Pol III specificity of vertebrate U6 transcription, by a mechanism for which the molecular details remain to be defined (Mattaj et al. 1988; Lobo and Hernandez 1989; Lobo et al. 1990; Lescure et al. 1991). A number of PSE-binding factors, termed SNAPc, PBP, and PTF, have been partially purified from human cells (Waldschmidt et al. 1991; Murphy et al. 1992; Simmen et al. 1992; Sadowski et al. 1993); it is not yet clear whether these factors are related to one another. Interestingly, the SNAPc complex contains TBP as well as several TAFs. SNAPc differs from the three TBP-TAF complexes mentioned above in that it is not polymerase-specific: It 
binds to the PSE in both Pol II- and Pol III-transcribed snRNA genes (Sadowski et al. 1993).

In the plant Arabidopsis, the structure of snRNA genes and the mechanism of polymerase specificity have diverged from the vertebrate genes. The promoters of the plant U2 and U6 genes that are transcribed by Pol II and Pol III, respectively, each comprise the same two upstream promoter elements, a sequence termed USE and a TATA box (Waibel and Filipowicz 1990a). The spacing between these elements differs, and this difference determines polymerase specificity (Waibel and Filipowicz 1990b). The mechanism by which polymerase specificity is mediated by the distance between two elements is as yet unclear.

Yeast snRNA promoters have diverged from those of the vertebrate and plant genes. A similarity is that Pol III transcribes the yeast U6 gene (Moenne et al. 1990) using a combination of elements found in both Pol II and Pol III genes. Yeast U6 contains a binding site for TFIIIC downstream of the transcription start site, as well as an upstream promoter containing a -30 TATA element (Brow and Guthrie 1990). Sequences upstream of the yeast U6 TATA box have no apparent role in transcription of the gene in vivo or in vitro (Burnol et al. 1993a; Eschenlauer et al. 1993). In tRNA genes, TFIIIC-binding sites consist of two intragenic consensus elements termed A and B blocks (for review, see Geiduschek and Tocchini-Valentini 1988). The TFIIIC site in the U6 gene consists of a nonconsensus intragenic A block and a consensus B block at an unusually distant location 234-bp downstream from the transcription start site (Brow and Guthrie 1990; Burnol et al. 1993a). The yeast U6 promoter therefore differs from tRNA promoters in the inclusion of a TATA element and in the location and strength of its TFIIIC site. There are some discrepancies in previous work in the requirements for the U6 TFIIIC and TATA elements between different transcription systems. Although the TATA element is required for maximal levels of transcription and accurate start site positioning in vivo and is essential for U6 transcription in vitro, it is not essential in vivo, possibly reflecting a redundant function performed by the A block (Burnol et al. 1993a; Eschenlauer et al. 1993). This is reminiscent of the nonessentiality of some Pol II TATA boxes in vivo, where the initiator element at the transcription start site can perform a redundant function (Hen et al. 1982; Dierks et al. 1983; Smale and Baltimore 1989|. The TFIIIC site is necessary for transcription in vivo but is dispensable in relatively purified in vitro systems (Margottin et al. 1991; Burnol et al. 1993a; Eschenlauer et al. 1993; Joazeiro et al. 1994). In one study the in vivo function of TFIIIC was suggested to involve counteracting chromatin-mediated repression, as TFIIIC relieved inhibition of U6 transcription by nucleosomes assembled in vitro (Burnol et al. 1993b).

Here, we examine how juxtaposition of a TATA-containing promoter and a TFIIIC site determines Pol III specificity of yeast U6 transcription. Polymerase specificity is presumably determined at an early stage of preinitiation complex formation. Transcription complex assembly on tRNA genes consists of TFIIIC-mediated recruitment of TFIIIB to DNA upstream of the transcription start site, prior to the binding of Pol III (Kassavetis et al. 1990). The same purified components of TFIIIB (TBP, BRF1, and at least one other factor, termed TFIIIB90 or $\left.B^{\prime \prime}\right)$ are required for both U6 and tRNA transcription in vitro (Huet et al. 1994; Joazeiro et al. 1994). Two models that could explain Pol III specificity of yeast U6 invoke recruitment of TFIIIB by Pol III-specific promoter sequences. In the first model, sequences flanking the U6 TATA box would encode a preference for binding of TFIIIB compared with Pol II factors. In this model TFIIIC would function to enhance transcription from this basal Pol III-specific promoter. This model is consistent with the in vitro experiments mentioned above, in which the upstream promoter of U6 was sufficient to direct transcription by Pol III in the absence of the TFIIIC site (Margottin et al. 1991; Burnol et al. 1993a; Joazeiro et al. 1994). In the second model, polymerase specificity would be determined by TFIIIC, which would recruit TFIIIB to the U6 TATA element. In this model the TATA box would serve to compensate for a weak TFIIICbinding site. To test these models we examined the binding of Pol II and Pol III factors to the upstream promoter of U6 in vitro. To determine U6 polymerase specificity in vivo, hybrid U6 derivatives containing Pol III and Pol II tandem transcription start sites were constructed and introduced into yeast strains deficient for Pol II or Pol III transcription. Our data support the second model, because we find that the upstream promoter of U6 is not sufficient to encode Pol III specificity in vitro or in vivo, and Pol III specificity is determined by TFIIIC.

\section{Results}

\section{BRF1 and TFIIB bind comparably to the upstream yeast U6 promoter and to Pol II promoters in vitro}

We tested a model in which the yeast U6 upstream promoter specifically binds Pol III factors in vitro, whereas a TATA-containing promoter of a Pol II gene would encode specificity for binding Pol II factors. Because polymerase specificity is presumably determined at an early stage of preinitiation complex formation, we compared the binding of factors that normally interact directly with TBP and polymerase in Pol III and Pol II preinitiation complexes. These factors, BRF1 and TFIIB, share sequence homology and may serve analogous roles in Pol III and Pol II initiation, respectively (Buratowski and Zhou 1992; Colbert and Hahn 1992; Lopez-De-Leon et al. 1992). It is not clear whether TFIIIB binds to the promoter as a multisubunit complex (of TBP, BRF1, and TFIIIB90) in vivo, or by stepwise assembly of these components as defined in order of addition experiments in vitro (Kassavetis et al. 1992). Stepwise assembly was implied from the lack of evidence for a stable multisubunit TFIIIB complex (Huet et al. 1994). TBP was bound to a probe spanning from -82 to +33 relative to the U6 transcription start site, and binding of BRF1 or TFIIB to these U6 TATA/TBP complexes was assessed in gel shift 
assays. TBP bound as well to the U6 TATA box as to a number of Pol II TATA-containing promoters tested (Fig. 1, lane 1). Surprisingly, BRF1 bound as well to TATA/ TBP complexes assembled on the promoters of the yeast Pol II-transcribed CYC1 and HIS4 genes, as it did to U6 TATA/TBP complexes (lanes 2-5). Likewise, TFIIB bound as well to the $\mathrm{U} 6$ promoter as it did to the CYC1 and HIS4 promoters (lanes 6-8). Binding of BRF1 compared with TFIIB to individual promoters occurred within a fivefold range of affinity (lanes 2-5 compared with lanes $6-8$ ). Because the concentration of active protein within the recombinant TFIIB or BRF1 preparations is unknown, it is most informative to compare binding of the same factor to different promoters. Such a comparison reveals no specificity in binding of BRFl or TFIIB to promoters of genes specifically transcribed by Pol III or Pol II. Thus, the U6 promoter behaves like the Pol II promoters in its binding to BRF1 and TFIIB. Likewise, a complex of all three TFIIIB components, TBP, BRF1, and TFIIIB90, exhibited no specificity between the U6 and Pol II promoters (S. Miller, unpubl.). These results raise the question of how polymerase specificity is determined at the U6 promoter as well as at TATA-containing Pol II promoters.

\section{Transcription of the upstream promoter of yeast U6 is not Pol III-specific in vivo or in vitro}

We sought to determine the polymerase specificity of the U6 upstream promoter in vivo. A hybrid gene was constructed in which the yeast U6 promoter including the TATA element and transcription start site, was juxtaposed with the start site of a Pol II gene. The U6 promoter from -81 to +25 was fused to the +13 start site and downstream coding sequence of the yeast HIS 3 gene (U6/HIS3; Fig. 2a). The two start sites in this construct were situated with respect to the U6 TATA box at distances appropriate for Pol III and Pol II in yeast. The HIS3 gene from -59 through its coding region was employed as a Pol II-specific control (HIS3; Fig. 2A). These genes were introduced on centromeric vectors into a temperature-sensitive yeast strain defective for Pol II transcription at the restrictive temperature of $37^{\circ} \mathrm{C}$, because of a mutation in the RPB1 gene (Nonet et al. 1987). S1 mapping of RNA derived from the temperature-sensitive transformants revealed that the upstream promoter of U6 is Pol II-specific in vivo (Fig. 2B). Transcription initiated at the Pol II start site and, like that of the Pol II control gene, was inhibited upon shifting the strain to $37^{\circ} \mathrm{C}$. Transcription was weak from both the U6/HIS3 promoter and the HIS3 promoter, as expected for basal level Pol II transcription in yeast. As indicated, transcription of U6/HIS3 did not initiate detectably at the Pol III start site. As a control, an unstable precursor tRNA mapped using an intron-specific probe (Cormack and Struhl 1992) was not reduced upon heat shock (not shown); furthermore, no inhibition of Pol III transcription occurred upon heat shock of this strain as shown below in Figure 5B (lanes 3-7 and lanes 8-12). These results were corroborated using two yeast strains, each containing a mutation in TBP that renders the strain specifically deficient for Pol III transcription (Cormack and Struhl 1993). As deduced by analysis of an unstable tRNA precursor, Pol III transcription was severely reduced in these strains when compared with the isogenic wild-type strain (not shown). In contrast, the U6/HIS3 gene was transcribed equally well in the wild-type and Pol III-deficient strains (see below, Fig. 6B, right).

As further confirmation of the Pol II specificity of the U6 promoter, we tested whether transcription of U6/ HIS3 could be stimulated by a Pol II enhancer element. The upstream activating sequence (UAS) of the yeast CYC1 gene fused upstream of the U6 promoter specifically activated transcription initiated from the Pol II start site (UAS/U6/HIS3; Fig. 3A,B, lanes 1-3). Transcription was inhibited at the restrictive temperature with the same kinetics exhibited by the UAS/HIS3 promoter (Fig. 3B, lanes 6-15). As expected from the results in Figure 2, no transcription was detected at the Pol III start site. The data in Figures 2 and 3 demonstrate for-
Figure 1. BRFl or TFIIB bind with comparative affinities to the upstream promoter of yeast U6 and to TATA-containing Pol II promoters. A gel retardation experiment is shown, in which $5 \mathrm{nM}$ of the conserved portion of TBP was incubated with the U6, CYCl, and HIS4 probes in conjunction with the indicated concentrations of recombinant BRF1 or yeast TFIIB. The positions of free probe and protein-DNA complexes are marked by arrows.
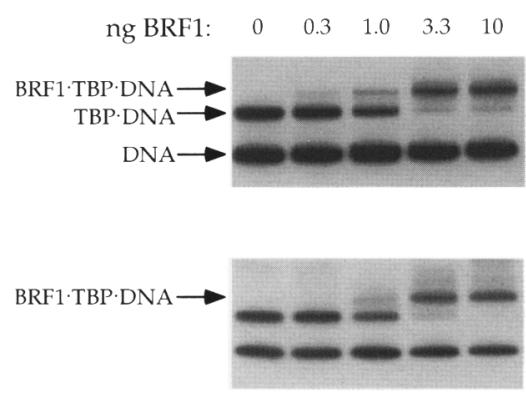

U6
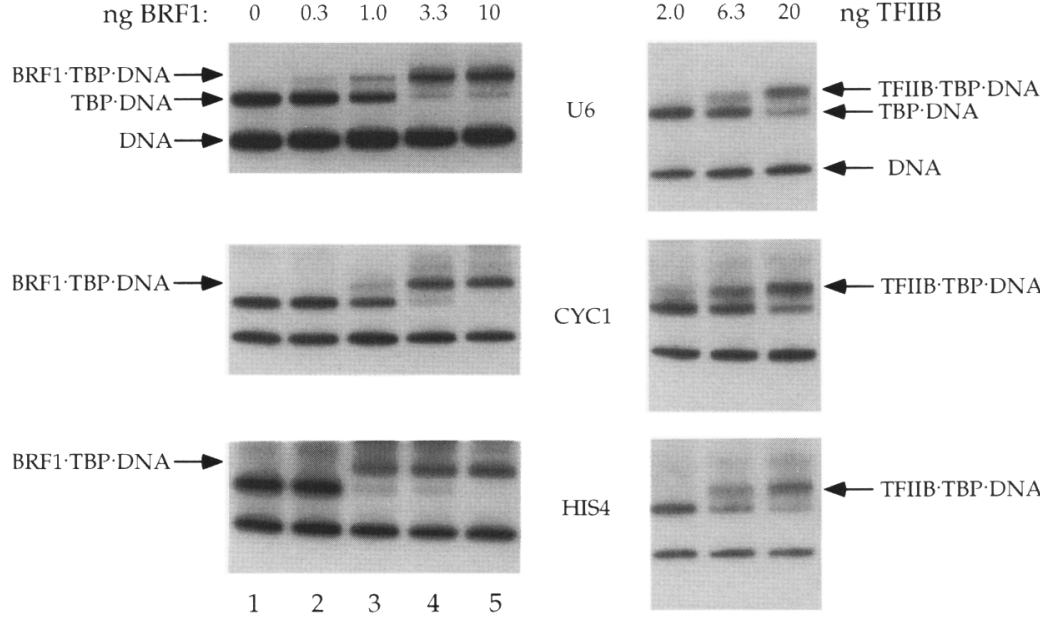

HIS4
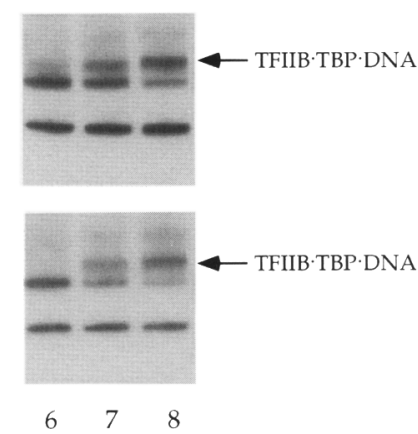
A

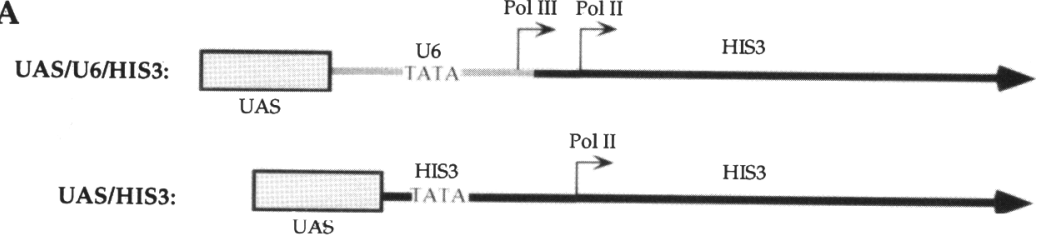

B

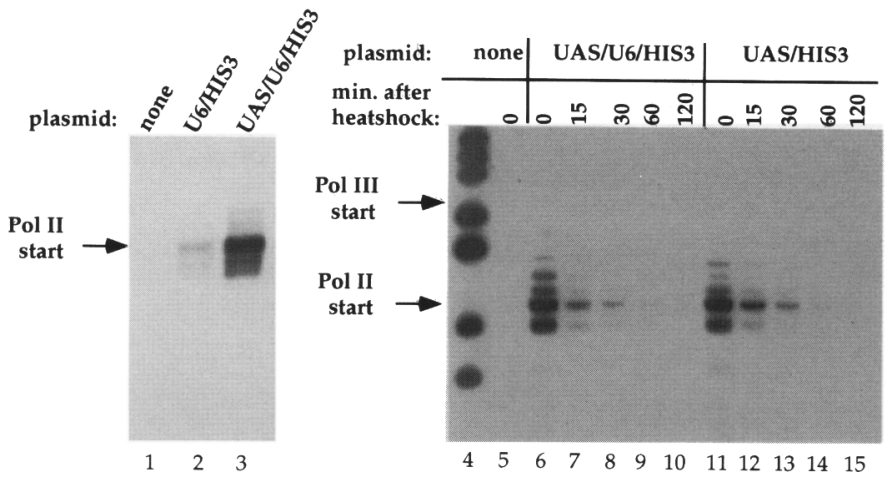

Figure 2. The upstream promoter of yeast U6 in pU6/HIS3 is Pol II-specific in vivo. ( $A$ ) Reporter constructs assayed in $B$. All constructs were derivatives of pRS315 (Sikorski and Hieter 1989). Solid and shaded lines designate HIS3 and U6 sequences, respectively; arrows represent transcription start sites. pU6/HIS3 contains the U6 promoter from -81 to +25 fused to the HIS 3 gene from +3 through the coding region. The $\mathrm{U} 6+1$ and HIS $3+13$ start sites were situated 30 - and 70-bp downstream of the U6 TATA box, respectively. pHIS 3 spans from -59 relative to its +1 start site through its coding region to +758 . To prevent polymerases reading into the promoters from vector sequences, all plasmids included the $U R A 3$ transcription terminator upstream of the sequences shown. $(B)$ Sl mapping of RNA from a temperature-sensitive strain containing the $r p b 1-1$ mutation and bearing pU6/HIS3 or pHIS3. Transformants were shifted to $37^{\circ} \mathrm{C}$, and samples were harvested at the times indicated. Arrows indicate transcription from the Pol II start site and the lack of detectable transcription from the Pol III start site. mation of a Pol II initiation complex at the U6 promoter in vivo. Such a complex might not be productive for transcription in the absence of the Pol II start site present in the hybrid U6/HIS3 gene, because sequences around the start site could influence selection of polymerase. However, the point of the hybrid gene was to determine whether the upstream portion of the U6 promoter, including its own Pol III start site, is Pol III-specific in vivo; clearly it is not.

An analogous template containing a GAL4 UAS was constructed and transcribed in vitro in a yeast nuclear extract $\left(\mathrm{UAS}_{\mathrm{G}} / \mathrm{U} 6 / \mathrm{HIS} 3\right.$; Fig. 4). Basal transcription in the extract was detected at both the Pol II and Pol III start sites and was very weak (lane 3). Consistent with the in vivo data above, addition of the GAL4-VP16 protein activated transcription initiated at the Pol II start site, which was inhibited by $10 \mu \mathrm{g} / \mathrm{ml}$ of $\alpha$-amanitin as expected for Pol II transcription (lanes 6,7). Upon addition of $12 \mathrm{nM} \mathrm{BRF1}$ to the extract, initiation at the Pol III start site was detected (lane 4). Transcription from this start site was resistant to $1 \mu \mathrm{g} / \mathrm{ml}$ of $\alpha$-amanitin as expected for Pol III transcription (lane 5). When GAL4-VP16 was prebound to the $\mathrm{UAS}_{\mathrm{G}} / \mathrm{U} 6 / \mathrm{HIS} 3$ template, transcription in the BRF1-supplemented nuclear extract occurred from both the Pol II and the Pol III start sites (lane 8). Pol II transcription was inhibited two- to threefold by BRF1 (lane 6 vs. 8). Transcription at only the Pol II start site was inhibited by $1 \mu \mathrm{g} / \mathrm{ml}$ of $\alpha$-amanitin (lane 9). This
U6/HIS3:
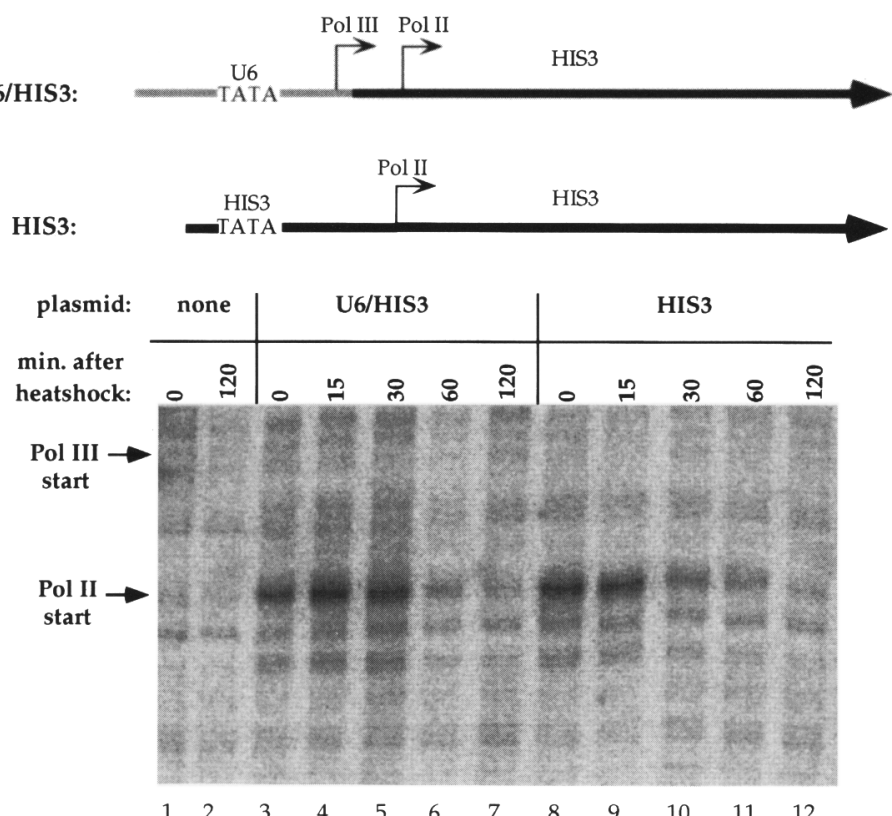

Figure 3. Transcription from the yeast U6 promoter is activated by a Pol II UAS. (A) Reporter constructs used in $B$. pUAS/U6/HIS3 and pUAS/ HIS3 are equivalent to pU6/HIS3 and pHIS 3 described in Fig. 2, except for the addition of the $C Y C 1$ UAS upstream of the promoter. $(B)$ (Lanes 1-3) S1 mapping experiment to compare transcription from the U6 promoter \pm a UAS. pU6/ HIS3 is described in Fig 2. Cells bearing the indicated plasmids were grown at the permissive temperature. (Lane 4) DNA size standards, MspIdigested pBR322. (Lanes 5-15) A primer extension assay of RNA from heat-shocked $r p b 1-1$ cells bearing the indicated plasmids. 


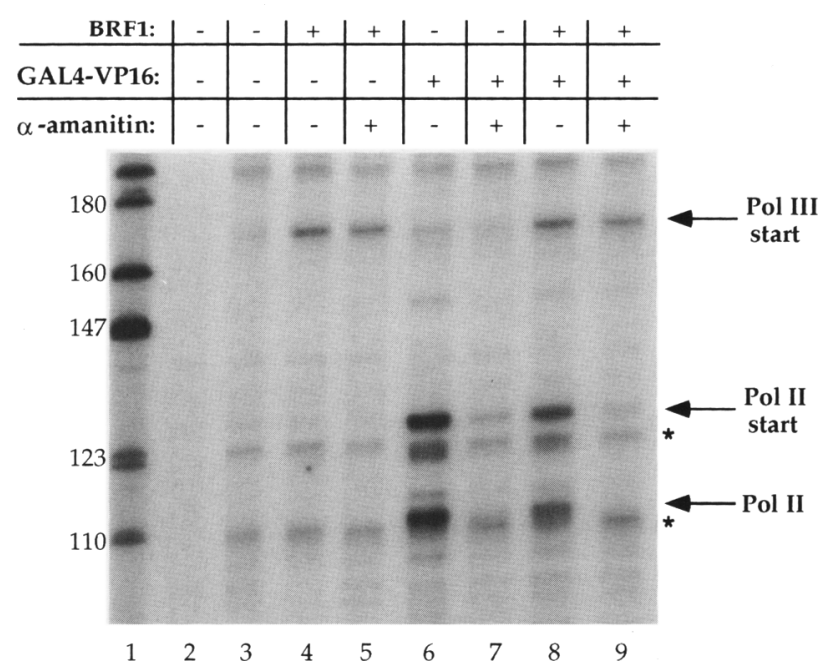

Figure 4. Dual polymerase specificity of the yeast U6 promoter in vitro. Primer extension mapping of transcription of the hybrid U6 template in a yeast nuclear extract. The template, $\mathrm{pUAS}_{\mathrm{G}} / \mathrm{U} 6 / \mathrm{HIS} 3$, is equivalent to pUAS/U6/HIS3 (Fig. 3), except that the UAS was derived from the GAL4 gene. DNA size standards are shown in lane 1 . In lane 2, no template was included. Where indicated, reactions contained GAL4-VP16 protein prebound to the template for $20 \mathrm{~min}$ prior to addition of other factors, BRFl at a final concentration of $12 \mathrm{nM}$ and $\alpha$-amanitin at $10 \mu \mathrm{g} / \mathrm{ml}$. Arrows indicate transcription induced by BRF1 and GAL4-VP16 from the Pol III and the Pol II start sites, respectively. Some background bands that appear to be derived from elsewhere on the template are designated by an asterisk; these bands were not produced when transcription was mapped using a different primer (not shown) and were unaffected by addition of $1 \mu \mathrm{g} / \mathrm{ml}$ of $\alpha$-amanitin, GAL4-VP16, or BRF1.

dual polymerase specificity is consistent with the data in Figure 1, which revealed binding of both BRF1 and TFIIB to the U6 promoter. These in vitro results suggest further that the lack of detectable Pol III transcription from the U6/HIS3 template in vivo may be a consequence of limiting concentrations of BRF1. One possibility is that in vivo concentrations of BRF1 are inadequate to compete with Pol II factors for binding to the U6/HIS3 template, because we have observed competition between Pol II and Pol III factors in vitro: When GAL4-VP16 was not prebound to the template but, rather, was added simultaneously with BRF1, transcription occurred from both start sites, but transcription at the Pol II start site was inhibited at least fivefold by BRF1 (not shown).

\section{A TFIIIC-binding site converts the upstream U6 promoter to a Pol III promoter}

Because the above results showed that the upstream promoter of U6 is not Pol III-specific in vivo, we tested whether the TFIIIC-binding site located downstream of the U6 start site in the wild-type gene determines Pol III specificity. To test this, a TFIIIC-binding site was inserted downstream of the U6/HIS3 promoter (Fig. 5A:
U6/HIS3/TFIIIC). A TFIIIC-binding site in a tRNA gene consists of two conserved elements, termed A and B blocks, separated by at least $31 \mathrm{bp}$ /Geiduschek and Tocchini-Valentini 1988). The inserted TFIIIC site comprised U6 sequences from +21 to +310 relative to the U6 start site, encompassing the intragenic A block and downstream B block. The intervening Pol II start site prevented the TFIIIC site from being positioned at the wild-type distance from the U6 start site. The A and B blocks were located 63 and 276 bp from the Pol III start, respectively, compared with 21 and $234 \mathrm{bp}$ in the wildtype U6 promoter. Strikingly, the TFIIIC site converted the hybrid promoter to a predominantly Pol III promoter (Fig. 5B). The TFIIIC element promoted usage of the Pol III start site (lane 3). Furthermore, these transcripts were not reduced upon shifting the Pol II temperature-sensitive strain to the restrictive temperature (lanes 3-7). RNA initiated at the U6 start site was therefore synthesized by Pol III, as evidenced further by its decrease in the Pol III-defective strains (lanes 15-17). For reasons we do not understand, transcription at the Pol II start site was more heterogeneous than in the absence of the TFIIIC site. RNA initiated at the Pol II start site was reduced by twofold in the Pol II temperature-sensitive strain and unaffected in the Pol III-defective strains, so we presume that at least a proportion of these transcripts was synthesized by Pol II.

Because the TFIIIC element activated Pol III transcription of the U6/HIS3 gene while the Pol II enhancer activated Pol II transcription, we tested the polymerase specificity of a gene in which both elements were combined. Surprisingly, the CYC1 UAS inserted upstream of the U6/HIS3/TFIIIC gene (Fig. 5A, UAS/U6/HIS3/TFIIIC) did not significantly stimulate transcription from either start site (Fig. 5B, lanes 8-12). These results show that the TFIIIC-binding site confers Pol III specificity to the U6 promoter, irrespective of the presence of a Pol II enhancer.

In the endogenous U6 gene, the TATA element is not absolutely essential in vivo but, rather, specifies correct initiation. The nonessential nature of the TATA box might reflect a redundant function performed by the $\mathrm{A}$ block (Eschenlauer et al. 1993). Because the distance between the A block and the U6 start site was suboptimal in the U6/HIS3/TFIIIC gene, this could increase the importance of the TATA box. Transcription initiated at the Pol III start site (and also at the Pol II start site) was abolished by a mutation in the U6 TATA box (Fig. 5B, lanes 13,14). Although the A block and TATA box may cooperate to direct initiation at the +1 start, the TATA box may specify start sites slightly farther upstream in the absence of the A block (Eschenlauer et al. 1993). 5' ends of RNA initiated at the Pol III start site of U6/HIS3/ TFIIIC were mapped between -2 and -4 (not shown). Thus, the A block may be nonfunctional in the U6/ HIS3/TFIIIC gene, although we cannot rule out the formal possibility of a pseudo-A-like element within these sequences. However, computer searches did not reveal sequences in an appropriate location bearing significant homology (less than four mismatches) to the A block 
A

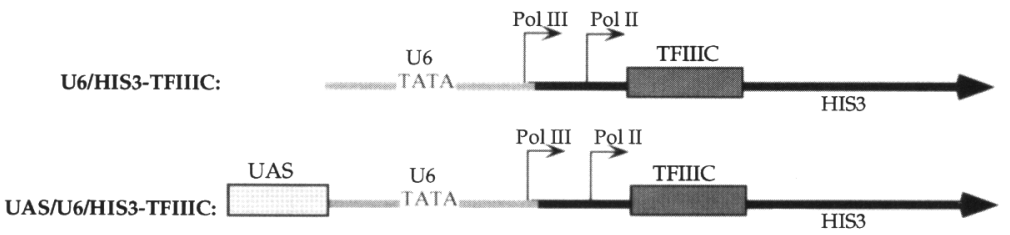

B

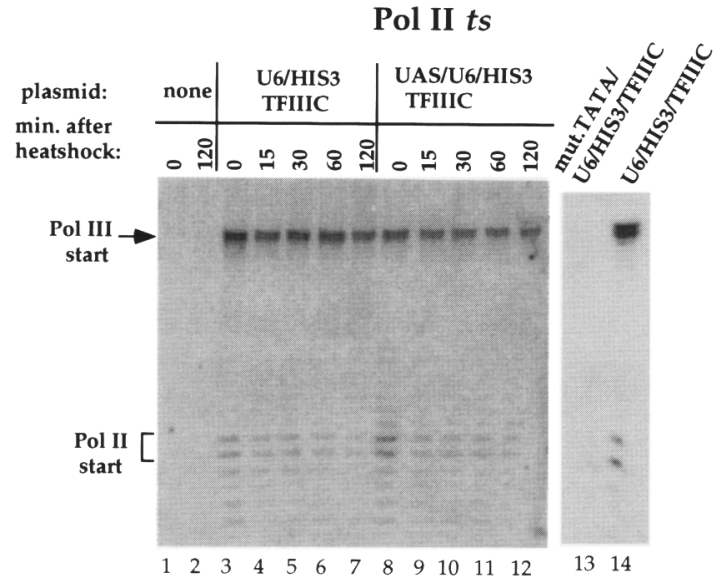

consensus (TrgennAgy/n)GG; Geiduschek and TocchiniValentini 1988).

The B block element is sufficient to determine POI III specificity of the U6/HIS3 gene

The above results suggested that the A block may not be functional in the U6/HIS3/TFIIIC gene. Because the A block and TATA box might play similar roles in positioning the initiation complex, the distant B block may have cooperated with the TATA box to transcribe U6/ HIS3/TFIIIC, albeit relatively weakly. We therefore
Figure 5. The TFIIIC site converts the yeast U6 promoter to Pol III specificity. $(A)$ DNA constructs used in $B$. Nomenclature is as described in Fig. 2A. pU6/HIS3TFIIIC and pUAS/U6/HIS3-TFIIIC are equivalent to pU6/HIS3 and pUAS/U6/ HIS3, respectively (Figs. 2 and 3), except for the TFIIIC-binding site. $(B)(L e f t) S 1$ protection assay of RNA from heat-shocked (lanes 1-12) and non-heat-shocked (lanes 13-14) $r p b 1-1$ cells bearing the indicated plasmids. The TATA mutation in lane 13 consisted of the sequence TAGAGA instead of TATAAA. (Right) (lanes 15-17) S1 mapping of RNA from yeast strains compromised for Pol III transcription attributable to the indicated point mutation in the TBP gene, and containing pU6/HIS3-TFIIIC. As controls, actin transcription was unaffected in the mutant strains whereas transcription of an unstable tRNA ${ }^{\text {Ile }}$ precursor was reduced (not shown).
A

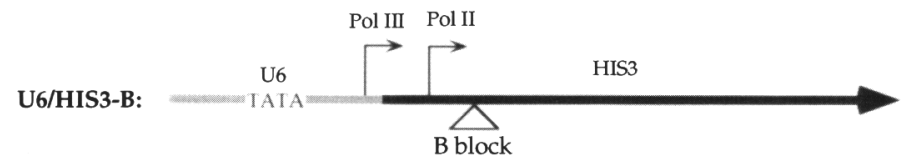

B

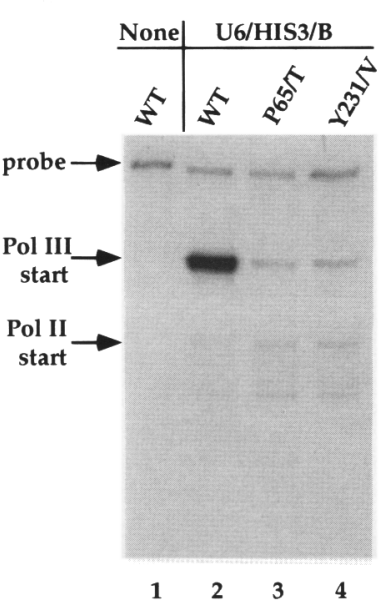

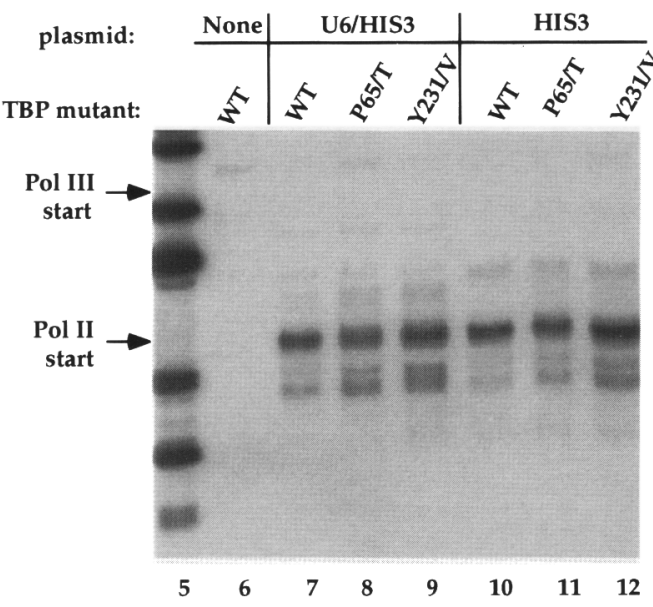

Figure 6. The B-block element converts the U6/HIS3 gene to Pol III specificity. $(A)$ DNA construct used in $B$. pU6/HIS3-B is equivalent to pU6/HIS3 (Fig. 2), except for the insertion of a tDNA ${ }_{3}^{\text {Leu-derived se- }}$ quence containing the 11-bp $B$ block flanked by 7 bp of tDNA sequence. $(B) \mathrm{S} 1$ protection of RNA from the Pol III-defective TBP mutant strains bearing pU6/ HIS3/B (lanes 2-4) or the indicated control plasmids described in Fig. 2, which did not contain the B block (lanes 7-12). Lanes 5-12 were exposed four times longer than lanes $1-4$, yet no initiation was detected at the Pol III start site of pU6/HIS3 in the absence of the B block, consistent with the data obtained using the Pol II temperaturesensitive strain (Fig. 2B). 
lacked a B block (U6/HIS3, lanes 7-9). Even at this longer exposure, transcription was not detected at the Pol III start site in the absence of the B block; furthermore transcription at the Pol II start site was not reduced in the Pol III-defective mutant strains as expected (lanes 7-9|. As a further Pol II-specific control, transcription from the HIS3 gene was not reduced in the mutant strains (lanes 10-12). It is noteworthy that transcription of U6/HIS3/B was at least fivefold more efficient than U6/HIS3/TFIIIC in which the B block was 206 bp farther from the start site. These results demonstrate that the B-block component of a TFIIIC-binding site is sufficient to convert the polymerase specificity of U6/HIS3 to Pol III.

\section{Discussion}

The role of TFIIIC in polymerase specificity

The yeast U6 promoter comprises an unusual combination of an upstream TATA-containing region and a suboptimal downstream TFIIIC-binding site. Here, we have distinguished between two models by which this combination of elements determines Pol III specificity of U6 transcription. In the first model, the upstream sequences specifically bind Pol III factors, compared with a Pol II promoter that would specifically bind Pol II factors. In the second model, TFIIIC determines the Pol III specificity of U6 transcription; in this model the upstream sequences would serve to increase the efficiency or accuracy of this Pol III-specific transcription. Contrary to the first model in which the upstream promoter of U6 should selectively recruit Pol III factors, we found that the U6 promoter behaved exactly like a Pol II promoter in its binding to Pol III and Pol II factors. We also established that the U6 upstream promoter is not Pol III-specific in vivo. Our results show that in contrast to the vertebrate and plant U6 genes, polymerase specificity in the yeast gene is not determined by the upstream promoter. Instead, our results confirm the second model, since we have shown that TFIIIC is the critical determinant of Pol III specificity of U6 transcription.

We speculate that in vivo, TFIIIC determines Pol III specificity of U6 transcription by assisting the binding of TFIIIB as occurs at other Pol III promoters which do not contain TATA elements. What, then, is the role of the U6 TATA box? On tRNA genes TFIIIB recruited by TFIIIC binds to DNA upstream of the transcription start site, in an essentially non-sequence-specific manner (Kassavetis et al. 1989, 1991). Nevertheless, 5'-flanking regions of tRNA genes, which in some cases contain TATA-like sequences, can also influence the efficiency of transcription (Geiduschek and Tocchini-Valentini 1988). The necessity of the U6 TATA element is most apparent under in vitro conditions, whereas in vivo it is mainly important for accurate positioning of the start site (Burnol et al. 1993a; Eschenlauer et al. 1993). This lack of absolute requirement for the TATA box in vivo may reflect a redundant function performed by the A block (Burnol et al. 1993a; Eschenlauer et al. 1993). In this respect, U6 is similar to Pol II promoters in which TATA mutations did not reduce initiation severely in vivo (Hen et al. 1982; Dierks et al. 1983), presumably because of a similar function mediated by an initiator element (Smale and Baltimore 1989; Zenzie-Gregory et al. 1992). The U6 TFIIIC-binding site is probably weak owing to its nonconsensus A block and distant B block. A model consistent with our results is that cooperative binding between TFIIIB centered at the TATA element and TFIIIC at its weak downstream site promotes proper positioning of TFIIIB to form a stable Pol III preinitiation complex (Fig. 7). The A block appears to be relatively weak because when changed into a consensus A block, U6 transcription was increased (Burnol et al. 1993a). Sec-

\section{(A) TATA-containing Pol II gene}

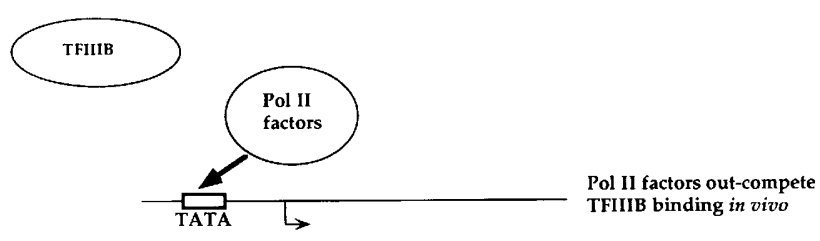

(B) TATA-containing U6 gene

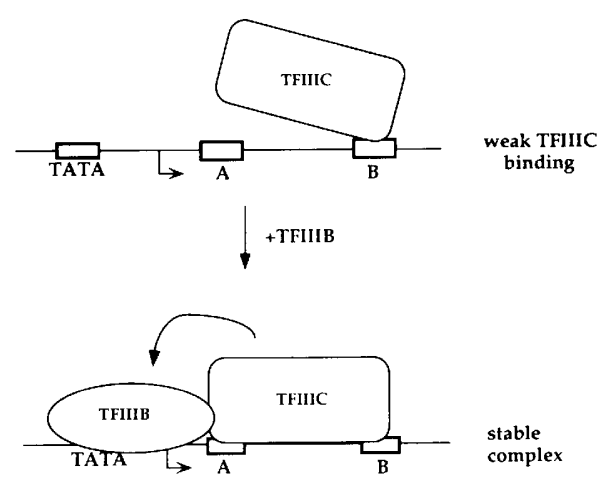

Figure 7. A schematic model for preinitation complex formation at the TATA-containing yeast U6 promoter. $|A|$ Binding of TFIIIB to a TATA-containing gene without a TFIIIC site would be relatively unstable in vivo, at least in part from competition by Pol II factors. $(B)$ The U6-binding site is probably weak because of its distal B block and nonconsensus A block. TFIIIC is designated as binding to the B block but not the A block, because a TFIIIC footprint was observed only over the B block on the U6 gene as well as on a tRNA gene with artifically separated A and B blocks (Fabrizio et al. 1987; Eschenlauer et al. 1993). TFIIIC would recruit TFIIIB to the upstream U6 promoter, where the TATA box may assist the weak TFIIIC-binding site in correct positioning of TFIIIB. Cooperative binding between TFIIIC and TFIIIB bound at the TATA box would promote stable Pol III preinitation complex formation and accurate initiation. Stable binding of Pol III factors to the promoter would effectively compete with the binding of Pol II factors. The topology of the DNA between the A and B blocks, which may be looped out to juxtapose the A and B blocks (Burnol et al. 1993a; Eschenlauer et al. 1993), is not indicated in the model. 
ond, a gene in which the B block was placed $70 \mathrm{bp}$ from the transcription start site (U6/HIS3/B), functioned at least fivefold more efficiently than one in which the $B$ block was $200 \mathrm{bp}$ farther downstream (U6/HIS3/TFIIIC).

In relatively purified in vitro transcription systems, the upstream U6 promoter lacking a TFIIIC-binding site can be transcribed by Pol III. In contrast, TFIIIC is necessary for U6 transcription in crude in vitro systems and in vivo (Margottin et al. 1991; Burnol et al. 1993a; Eschenlauer et al. 1993; Joazeiro et al. 1994). To reconcile these discrepancies, the in vivo function of TFIIIC was proposed to involve relief of chromatin-mediated repression, as TFIIIC counteracted inhibition of U6 transcription by nucleosomes assembled in vitro (Burnol et al. 1993b). A model in which TFIIIC directly facilitates binding of TFIIIB at the U6 promoter is also consistent with an additional role for TFIIIC in exclusion of nucleosomes or other non-nucleosomal inhibitory factors. TFIIIC-mediated recruitment of TFIIIB implies that the variable requirement for TFIIIC could also be explained at least partially on the basis of the relative abundance or activity of TFIIIB in these systems. Excess TFIIIB, which can bind directly to the U6 TATA box, might obviate the requirement for TFIIIC. In vitro systems in which TFIIIC is not required are optimized for Pol III but not Pol II transcription, whereas under more stringent conditions, that is, in crude in vitro systems and in vivo, the concentration or activity of Pol III factors could be limiting. This hypothesis is supported strongly by our data in which transcription by Pol III from the U6/HIS3 template lacking a TFIIIC site was almost undetectable in a yeast nuclear extract unless supplemented with BRF1 (Fig. 4).

\section{Binding of BRF1 to TATA-containing Pol II promoters}

BRF1 bound at least as stably as TFIIB to TBP/TATA complexes formed on classical Pol II promoters in our in vitro binding assays. This result is surprising, as Pol II promoters presumably do not bind BRF1 in vivo. Do such TBP/BRF1 complexes recruit the remaining Pol III factors and initiate transcription on Pol II promoters? We tested this by transcribing a number of Pol II templates in crude nuclear extracts, which, as shown in Figure 4, were limiting for BRF1. Upon addition of BRF1, we did not observe Pol III transcription of Pol II templates (not shown). Because stable binding of RNA polymerases might be influenced by sequences around the transcription start site, the binding of TFIIIB might not necessarily result in initiation.

Irrespective of whether binding of TFIIIB to Pol II TATA boxes leads to transcription, binding per se would presumably be inhibitory to Pol II transcription by competition with Pol II factors. In support of this, we have observed competition between Pol II and Pol III factors in vitro. GAL4-VP16-activated transcription was inhibited when BRF1 was added simultaneously with GAL4-VP16 to Pol II transcription reactions (not shown). The U6 promoter behaved similarly, because as mentioned in the Results, simultaneous addition of these proteins to the
$\mathrm{UAS}_{\mathrm{G}} / \mathrm{U} 6 / \mathrm{HIS} 3$ template caused strong inhibition of GAL4-VP16-activated transcription at the Pol II start site. Transcription was inhibited to a lesser extent (twoto threefold) when GAL4-VP16 was prebound to the U6 template (Fig. 4) or to Pol II templates (not shown). Competition between Pol II and Pol III factors has also been documented in vivo. In a previous study, yeast TBP mutants defective for Pol III transcription exhibited increased levels of Pol II transcription in vivo (Cormack and Struhl 1993). One explanation for this increase invokes limiting amounts of TBP. Alternatively, because these TBP mutants appeared to be compromised for interaction with BRF1 (Cormack and Struhl 1993), the increase in Pol II transcription could reflect relief of inhibition by BRF1. It is possible that certain factors reduce inappropriate binding of BRF1 to Pol II TATA boxes in vivo. The Pol II inhibitor Drl was shown recently to inhibit tRNA transcription, apparently by destabilizing the interaction of TBP with BRF1 (White et al. 1994). Perhaps Drl or, alternatively, Pol II transcription activators inhibit inappropriate binding of BRF1 to Pol II promoters. One postulated function of transcriptional activators is to block assembly pathways leading to the formation of nonproductive Pol II preinitiation complexes that can inhibit transcription (Hahn 1993). Perhaps activators also block formation of inhibitory Pol III preinitiation complexes.

\section{Inhibition of UAS-mediated Pol II activation by TFIIIC}

In our experiments, Pol II transcription from the U6 promoter occurred efficiently only in the absence of a TFIIIC-binding site. In the context of a TFIIIC site, a UAS no longer activated Pol II transcription from the U6 promoter (Fig. 5, UAS/U6/HIS3/TFIIIC). This could reflect Pol III factors out-competing binding of Pol II factors, even in the presence of the Pol II activator. An alternative model explaining the incompatibility between the UAS and TFIIIC site, which is consistent with our results, is that TFIIIC bound to intragenic sequences can present an obstacle to elongating Pol II complexes. Although TFIIIC is not required to remain bound to tRNA templates subsequent to assembly of TFIIIB in vitro (Kassavetis et al. 1990), it is not known whether TFIIIC actually remains bound during transcription of Pol III genes in vivo. TFIIIC was displaced from a tRNA gene template by Pol III under specific in vitro conditions; it is not known whether such displacement occurs in vivo or is an essential component of Pol III transcription (Bardeleben et al. 1994). Perhaps TFIIIC/DNA complexes can constitute a roadblock to elongating Pol II but not Pol III.

\section{Polymerase specificity in vertebrates and yeast}

In contrast to the evolutionary conservation in the structure of the U6 snRNA and in Pol III specificity of U6 transcription from vertebrates to yeast, there is considerable divergence in the elements that promote U6 ex- 
pression. From the data presented here, the mechanism of polymerase specificity in the yeast gene appears to differ from the vertebrate and plant systems, where polymerase specificity is mediated by sequences upstream of the start site. In a previous study, a region of the Xenopus U6 promoter comprising the TATA box and transcription start site functioned comparably well in vivo when flanked by either an upstream PSE element or a downstream TFIIIC site derived from a tRNA gene (Parry and Mattaj 1990|. Thus, an alternative possibility is that the vertebrate PSE element is functionally equivalent to the yeast TFIIIC site in assembly of TFIIIB or a component of TFIIIB onto the U6 TATA box.

\section{Materials and methods}

\section{Gel retardation assays}

Binding reactions were performed as described (Auble and Hahn 1993 ) except that $6 \%$ polyacrylamide gels contained $2.5 \%$ glycerol, $45 \mathrm{~mm}$ Tris, $46 \mathrm{~mm}$ boric acid, $1.25 \mathrm{mM} \mathrm{Na}_{2}$ EDTA, and 0.5 $\mathrm{mM}$ dithiothreitol at $\mathrm{pH} 8.3$ and were made with freshly prepared ammonium persulfate; running buffer contained $45 \mathrm{mM}$ Tris, $46 \mathrm{~mm}$ boric acid, and $1.25 \mathrm{mM} \mathrm{Na}_{2}$ EDTA at pH 8.3. Gel and running buffers for BRF1-TBP- and TFIIB-TBP-binding reactions also contained $2.5 \mathrm{mM}$ or $1.5 \mathrm{mM} \mathrm{Mg}$ acetate, respectively. Gels were run at $4^{\circ} \mathrm{C}$ and $160 \mathrm{~V}$ without prerunning or cooling to $4^{\circ} \mathrm{C}$ prior to loading. The gels were loaded quickly while the gel was running. The TBP preparation, a truncated version lacking its nonconserved amino terminus, was kindly donated by J. Geiger (Yale University, New Haven, CT). Recombinant BRF1 was prepared as described (Colbert and Hahn 1992). Preparation of yeast TFIIB was described previously in Auble and Hahn (1993). The U6 probe spanned from -82 to +33 relative to the transcription start site of the SNR6 gene; the HIS4 probe consisted of sequences from -118 to +67 relative to the transcription start site; the CYC1 probe comprised sequences from -107 to +78 .

\section{In vitro transcription}

Yeast nuclear extract for in vitro transcription reactions was prepared essentially as described (Ponticelli and Struhl 1990). Transcription assays were also as in Ponticelli and Struhl (1990), except that reactions contained $2 \mu \mathrm{l}$ of nuclear extract comprising $80 \mu \mathrm{g}$ of protein, $300 \mathrm{ng}$ of $\mathrm{pUAS}_{\mathrm{G}} / \mathrm{U} 6 / \mathrm{HIS} 3$ template, and, where indicated, $12 \mathrm{nM} \mathrm{BRF1}, 10 \mu \mathrm{g} / \mathrm{ml}$ of $\alpha$-amanitin, or $23 \mathrm{ng}$ GAL4-VP16 fusion protein prebound to the template for $20 \mathrm{~min}$ prior to addition of other factors. Reactions were performed at room temperature for $30 \mathrm{~min}$. RNA was mapped by primer extension using a kinase-labeled oligonucleotide (his3D) of sequence 5'-CTTTTTCTGGGAAGATCGAGTGCTCTATCG homologous to the HIS3-coding region.

\section{DNA constructions}

A 400-bp StuI-SmaI fragment containing the terminator of the yeast $U R A 3$ gene was ligated to the SmaI site in the polylinker of pRS315 (Sikorski and Hieter 1989), from which an XhoI site had been removed by filling in ends with Klenow enzyme. The resulting vector, named pSL11, was used to construct the series of plasmids listed below.

pU6/HIS3 HIS3 sequences from +3 to +758 were PCR amplified with oligonucleotides that introduced $\mathrm{XbaI}$ and NotI sites at the $5^{\prime}$ and $3^{\prime}$ ends of the fragment, respectively. This fragment was inserted into corresponding sites located downstream of the terminator in pSL11. The U6 promoter from -81 to +25 was amplified by PCR using oligonucleotides that introduced BamHI and XhoI sites in tandem at the 5' end of the fragment and an $\mathrm{XbaI}$ site at the $3^{\prime}$ end. This fragment was ligated to the corresponding $B a m H I$ and $X b a I$ sites in the polylinker immediately $5^{\prime}$ of the HIS 3 sequences.

pHIS3 HIS3 sequences that promote initiation from the +1 start site were removed by truncating sequences upstream of -59 and mutation of bases -54 and -56 from $A$ and $T$ to $G$ and A, respectively. Bases -59 to +758 comprising the two point mutations were amplified using PCR oligonucleotides that introduced BamHI and XhoI sites in tandem at the $5^{\prime}$ end of the fragment and an $X b a I$ site at the $3^{\prime}$ end. This fragment was inserted into the $\mathrm{BamHI}$ and $\mathrm{XbaI}$ sites in the polylinker of pSL11.

pUAS/U6/HIS3 A SmaI-Xhol fragment comprising the CYC1 UAS from -312 to -178 , including subsites 1 and 2, was inserted into the SmaI and XhoI sites of pU6/HIS3 immediately $5^{\prime}$ of the U6 promoter, deleting the BamHI site.

pUAS/HIS3 The SmaI-XhoI UAS-containing fragment was ligated to the $S \mathrm{maI}$ and XhoI sites of pHIS3 immediately $5^{\prime}$ of the HIS3 promoter.

$p U A S_{G} / U 6 / H I S 3$ A SmaI fragment comprising the UAS of the yeast GAL4 gene was inserted into the SmaI site $5^{\prime}$ of the U6 promoter in pU6/HIS3.

pU6/HIS3/TFIIIC An NcoI site was introduced into pU6/ HIS3 at +27 of the HIS3-coding region by site-directed mutagenesis, to construct pU6/HIS3-Nco. Bases +21 to +310 of the U6 gene containing the TFIIIC site were PCR-amplified with $\mathrm{NcoI}$-containing oligonucleotides and ligated to the $\mathrm{NcoI}$ site of pU6/HIS3-Nco.

pmut.TATA/U6/HIS3/TFIIIC The U6 TATA box (TATAAATA) in pU6/HIS3/TFIIIC was replaced with the sequence TAGAGAAA by site-directed mutagenesis. The sequence of the mutagenic oligonucleotide (U6 TATA) was 5'-GAAAAAAACATTTTTCTCTAGTAGCCGAAAATAG.

pUAS/U6/HIS3/TFIIIC The XbaI-NotI fragment from pUAS/ U6/HIS3 was deleted and replaced with the XbaI-NotI fragment from $\mathrm{pU} 6 / \mathrm{HIS} 3 / \mathrm{TFIIC}$.

$p U 6 / H I S 3 / B$ Two annealed oligonucleotides flanked by $N c o$ I sites were inserted into the NcoI site at +27 of pU6/HIS3-Nco. The sequences of the oligonucleotides that were annealed prior to subcloning were 5'-CATGGATGCAAGGGTTCGAATCCCTTAGCAC (top) and 5'-CATGGTGCTAAGGGATTCGAACCCTTGCATC (bottom).

\section{In vivo temperature shifts and preparation of RNA}

Transformants were grown in $120 \mathrm{ml}$ of YPD to an $\mathrm{OD}_{600}$ of $0.5-0.8$ at the permissive temperature of $25^{\circ} \mathrm{C}$, preheat-shocked for $15 \mathrm{~min}$ at $37^{\circ} \mathrm{C}$, and returned to $25^{\circ} \mathrm{C}$ for $1 \mathrm{hr}$ prior to heat shock (Cormack and Struhl 1992). Cells were shifted to $37^{\circ} \mathrm{C}$, and $20 \mathrm{ml}$ of cells was removed at each time point, centrifuged, and used to prepare RNA. In Figure 6, the cells were harvested without heat shock as the Pol III-specific TBP mutant strains, 
although more compromised for growth at $37^{\circ} \mathrm{C}$ than at $30^{\circ} \mathrm{C}$, exhibited severe defects in Pol III transcription at $30^{\circ} \mathrm{C}$.

To prepare total cell RNA, pellets were resuspended in $0.6 \mathrm{ml}$ of RNA extraction buffer $(0.1 \mathrm{M} \mathrm{LiCl}, 0.1 \mathrm{M}$ Tris at $\mathrm{pH} 7.5,1 \mathrm{~mm}$ EDTA) and mixed with $0.3 \mathrm{ml}$ of glass beads and $16 \mu \mathrm{l}$ of $10 \%$ SDS. The resultant mixture was phenol-chloroform extracted twice, chloroform extracted once, and ethanol precipitated, and the RNA pellet was resuspended in $25 \mu \mathrm{l}$ of $\mathrm{H}_{2} \mathrm{O}$. This method typically yielded $50-100 \mu \mathrm{g}$ of RNA, of which $10 \mu \mathrm{g}$ was used for RNA mapping.

\section{Probes for RNA mapping}

RNA was mapped by $S 1$ protection in all figures except in lanes 4-15 of Figure 3b and in Figure 4, where RNA was mapped by primer extension using as a probe the his $3 \mathrm{D}$ oligonucleotide described above. For S1 protection, probes were derived from pU6/HIS3, pU6/HIS3-TFIIIC and pU6/HIS3-B. Probes were single-stranded and $5^{\prime}$-end-labeled, and spanned from -81 relative to the $\mathrm{U} 6$ start site, to $+138,+28$, and +168 relative to the HIS3 + 12 start site in U6/HIS3, U6/HIS3/TFIIIC, and U6/ HIS3/B, respectively. Probes were synthesized by linear PCR: Plasmids were linearized at the $X h o I$ site, and $1 \mu \mathrm{g}$ was used as a template in a $100-\mu 1$ PCR reaction with $1 \mu \mathrm{g}$ of kinase-labeled oligonucleotide. Oligonucleotides used for linear PCR were his3D (above) except in the case of U6/HIS3/TFIIIC, where the sequence of the oligonucleotide (hisNcoIIIC) was 5'-TGTCCACCATGGTCATCTTTGCC. PCR reactions also contained $200 \mu \mathrm{M}$ dNTPS, $1.5 \mathrm{mM} \mathrm{MgCl}_{2}, 5$ units of Taq polymerase, and buffer supplied by Promega. The reaction mixture minus polymerase was heated for $5 \mathrm{~min}$ at $94^{\circ} \mathrm{C}$, polymerase was then added, and PCR was performed using 3 cycles at $94^{\circ} \mathrm{C}, 40^{\circ} \mathrm{C}$, and $72^{\circ} \mathrm{C}$ for 40,60 , and $20 \mathrm{sec}$, respectively, followed by 22 cycles of $94^{\circ} \mathrm{C}, 50^{\circ} \mathrm{C}$, and $72^{\circ} \mathrm{C}$ for 40,30 , and $20 \mathrm{sec}$, respectively. Probes were purified on $5 \%$ polyacrylamide-TBE gels, eluted for at least $3 \mathrm{hr}$ at $37^{\circ} \mathrm{C}$ in $350 \mu \mathrm{l}$ of $0.5 \mathrm{M}$ ammonium acetate, $0.1 \%$ SDS, and $1 \mathrm{mM}$ EDTA and then were ethanol precipitated with $10 \mu \mathrm{g}$ of glycogen as carrier. Typical yields were $3 \times 10^{6} \mathrm{cpm}$, of which $10^{5} \mathrm{cpm}$ was hybridized to $10 \mu \mathrm{g}$ of total cell RNA per lane and start sites were mapped by $\mathrm{S} 1$ protection.

\section{Acknowledgments}

We thank B. Cormack, K. Struhl, and L. Stargell for TBP mutant strains, N. Woychik for the Pol II temperature-sensitive strain, Sally Lee for pSL11, and Stephen Miller for communication of unpublished data. We are also grateful to $M$. Groudine, R. Reeder, and members of our laboratory for comments on the manuscript. This work is supported by a Human Frontier Science Program fellowship to S.R., a U.S. Public Health Service training grant to T.C., and by a grant from the National Institutes of Health and a Leukemia Society Scholar Award to S.H.

The publication costs of this article were defrayed in part by payment of page charges. This article must therefore be hereby marked "advertisement" in accordance with 18 USC section 1734 solely to indicate this fact.

\section{References}

Auble, D.T. and S. Hahn. 1993. An ATP-dependent inhibitor of TBP binding to DNA. Genes \& Dev. 7: 844-856.

Baker, R.B., S. Camier, A. Sentenac, and B.D. Hall. 1987. Gene size differentially affects the binding of yeast transcription factor $\tau$ to two intragenic regions. Proc. Natl. Acad. Sci. 84: 8768-8772.
Bardeleben, C., G.A. Kassavetis, and G. Geideschek. 1994. Encounters of Saccharomyces cerevisiae RNA polymerase III with its transcription factors during RNA chain elongation. J. Mol. Biol. 235: 1193-1205.

Brow, D.A. and C. Guthrie. 1990. Transcription of a yeast U6 snRNA gene requires a polymerase III promoter element in a novel position. Genes \& Dev. 4: 1345-1355.

Buratowski, S. and H. Zhou. 1992. A suppressor of TBP mutations encodes an RNA polymerase III transcription factor with homology to TFIIB. Cell. 71: 221-230.

Burnol, A.F., F. Margottin, P. Schultz, M.-C. Marsolier, P. Oudet, and A. Sentenac. 1993a. Basal promoter and enhancer element of yeast U6 snRNA gene. J. Mol. Biol. 233: 644-658.

Burnol, A., F. Margottin, J. Huet, G. Almouzni, M. Prioleau, M. Mechali, and A. Sentenac. 1993b. TFIIIC relieves repression of U6 snRNA transcription by chromatin. Nature 362: 475477.

Colbert, T. and S. Hahn. 1992. A yeast TFIIB-related factor involved in RNA polymerase III transcription. Genes \& Dev. 6: 1940-1949.

Cormack, B.P. and K. Struhl. 1992. The TATA-binding protein is required for transcription by all three nuclear RNA polymerases in yeast cells. Cell 69: 685-696.

. 1993. Regional codon randomization: Defining a TATAbinding protein surface required for RNA polymerase III transcription. Science 262: 244-248.

Dierks, P., A. van Ooyen, M.D. Cochran, C. Dobkin, J. Reiser, and C. Weissmann. 1983. Three regions upstream from the cap site are required for efficient and accurate transcription of the rabbit beta-globin gene in mouse $3 \mathrm{~T} 6$ cells. Cell 32: 695-706.

Eschenlauer, J.B., M.W. Kaiser, V.L. Gerlach, and D.A. Brow. 1993. Architecture of a yeast U6 RNA gene promoter. Mol. Cell. Biol. 13: 3015-3026.

Fabrizio, P., A. Coppo, P. Fruscoloni, P. Benedetti, G. Di Segni, and G.P. Tocchini-Valentini. 1987. Comparative mutational analysis of wild-type and stretched tRNAleu ${ }_{3}$ gene promoters. Proc. Natl. Acad. Sci. 84: 8763-8767.

Geiduschek, E.P. and G.A. Kassavetis. 1992. RNA polymerase III transcription complexes. In Transcriptional regulation, pp. 247-280. Cold Spring Harbor Laboratory Press, Cold Spring Harbor, New York.

Geiduschek, E.P. and G.P. Tocchini-Valentini. 1988. Transcription by RNA polymerase III. Annu. Rev. Biochem. 57: 873914.

Hahn, S. 1993. Structure(?) and function of acidic transcription activators. Cell 72: 481-484.

Hen, R., P. Sassone-Corsi, J. Corden, M.P. Gaub, and P. Chambon. 1982. Sequences upstream from the T-A-T-A box are required for efficient transcription from the adenovirus serotype 2 major late promoter. Proc. Natl. Acad. Sci. 79: 7132-7136.

Hernandez, N. 1992. Transcription of vertebrate snRNA genes and related genes. In Transcriptional regulation, pp. 281313. Cold Spring Harbor Laboratory Press, Cold Spring Harbor Laboratory, New York.

- 1993. TBP, a universal eukaryotic transcription factor? Genes \& Dev. 7: 1291-1308.

Huet, J., C. Conesa, N. Manaud, N. Chaussivert, and A. Sentenac. 1994. Interactions between yeast TFIIIB components. Nucleic Acids Res. 22: 3433-3439.

Joazeiro, C.A.P., G.A. Kassavetis, and E.P. Geiduschek. 1994. Identical components of yeast transcription factor IIIB are required and sufficient for transcription of TATA box containing and TATA-less genes. Mol. Cell. Biol. 14: 27982808 . 
Kassavetis, G.A., D.L. Riggs, R. Negri, L.H. Nguyen, and E. Geiduschek. 1989. Transcription factor IIIB generates extended DNA interactions in RNA polymerase III transcription complexes on tRNA genes. Mol. Cell. Biol. 9: 2551-2566.

Kassavetis, G.A., B.R. Braun, L.H. Nguyen, and E.P. Geiduschek. 1990. S. cerevisiae TFIIIB is the transcription initiation factor proper of RNA polymerase III, while TFIIIA and TFIIIC are assembly factors. Cell 60: 235-245.

Kassavetis, G.A., B. Bartholomew, J.A. Blanco, T.E. Johnson, and E.P. Geiduschek. 1991. Two essential components of the Saccharomyces cerevisiae transcription factor TFIIIB: Transcription and DNA-binding properties. Proc. Natl. Acad. Sci. 88: 7308-7312.

Kassavetis, G.A., C.A.P. Joazeiro, M. Pisano, E.P. Geiduschek, T. Colbert, S. Hahn, and J.A. Blanco. 1992. The role of the TATA-binding protein in the assembly and function of the multisubunit yeast RNA polymerase III transcription factor, TFIIIB. Cell 71: 1055-1064.

Lescure, A., P. Carbon, and A. Krol. 1991. The different positioning of the proximal sequence element in the Xenopus RNA polymerase II and III snRNA promoters is a key determinant which confers RNA polymerase III specificity. $\mathrm{Nu}$ cleic Acids Res. 19: 435-441.

Lobo, S.M. and N. Hernandez. 1989. A 7bp mutation converts a human RNA polymerase II snRNA promoter into an RNA polymerase III promoter. Cell 58: 55-67.

Lobo, S.M., S. Ifill, and N. Hernandez. 1990. Cis-acting elements required for RNA polymerase II and III transcription in the human U2 and U6 snRNA promoters. Nucleic Acids Res. 18: 2891-2899.

Lopez-De-Leon, A., M. Librizzi, K. Puglia, and I.M. Willis. 1992. PCF4 encodes an RNA polymerase III transcription factor with homology to TFIIB. Cell 71: 211-220.

Margottin, F., G. Dujardin, M. Gerard, J.-M. Egly, J. Huet, and A. Sentenac. 1991. Participation of the TATA factor in transcription of the yeast U6 gene by RNA polymerase C. Science 251: 424-426.

Mattaj, I.W., N.A. Dathan, H.D. Parry, P. Carbon, and A. Krol. 1988. Changing the RNA polymerase specificity of $U$ snRNA gene promoters. Cell 55: 435-442.

Moenne, A., S. Camier, G. Anderson, F. Margottin, J. Beggs, and A. Sentenac. 1990. The U6 gene of Saccharomyces cerevisiae is transcribed by RNA polymerase C (III) in vivo and in vitro. EMBO J. 9: 271-277.

Murphy, S., J.-B. Yoon, T. Gerster, and R. G. Roeder. 1992. Oct-1 and Oct-2 potentiate functional interactions of a transcription factor with the proximal sequence element of small nuclear RNA genes. Mol. Cell. Biol. 12: 3247-3261.

Nonet, M., C. Scafe, J. Sexton, and R. Young. 1987. Eucaryotic RNA polymerase conditional mutant that rapidly ceases mRNA synthesis. Mol. Cell. Biol. 7: 1602-1611.

Parry, H.W. and I.W. Mattaj. 1990. Positive and negative funtional interactions between promoter elements from different classes of RNA polymerase III-transcribed gene. EMBO /. 9: 1097-1104.

Ponticelli, A.S. and K. Struhl. 1990. Analysis of Saccharomyces cerevisiae his 3 transcription in vitro : Biochemical support for multiple mechanisms of transcription. Mol. Cell. Biol. 10: $2832-2839$.

Rigby, P.W.J. 1993. Three in one and one in three: It all depends on TBP. Cell 72: 7-10.

Sadowski, C.L., R.W. Henry, S.M. Lobo, and N. Hernandez. 1993. Targeting TBP to a non-TATA box cis-regulatory element: A TBP-containing complex activates transcription from snRNA promoters through the PSE. Genes \& Dev. 7: 1535-1548.
Sikorski, R.S. and P. Hieter. 1989. A system of shuttle vectors and yeast host strains designed for efficient manipulation of DNA in Saccharomyces cerevisae. Genetics 122: 19-27.

Simmen, K.A., R. Waldshmidt, J. Bernues, H.D. Parry, K.H. Seifart, and I.W. Mattaj. 1992. Proximal sequence element binding and species specificity in vertebrate U6 snRNA promoters. J. Mol. Biol. 223: 873-884.

Smale, S.T. and D. Baltimore. 1989. The "initiator" as a transcription control element. Cell 57: 103-113.

Waibel, F. and W. Filipowicz. 1990a. U6 snRNA genes of Arabidopsis are transcribed by RNA polymerase III but contain the same two upstream promoter elements as RNA polymerase II-transcribed U-snRNA genes. Nucleic Acids Res. 18: $3451-3458$.

1990b. RNA-polymerase specificity of transcription of Arabidopsis U snRNA genes determined by promoter element spacing. Nature 346: 199-202.

Waldschmidt, R., I. Wanandi, and K. Siefart. 1991. Identification of transcription factors required for the expression of mammalian U6 genes in vitro. EMBO J. 10: 2595-2603.

White, R.J., B.C.-E. Khoo, J.A. Inostroza, D.A. Reinberg, and S.P. Jackson. 1994. Differential regulation of RNA polymerases I, II, and III by the TBP-binding repressor Drl. Science 266: $448-450$.

Zenzie-Gregory, B., A. O'Shea-Greenfield, and S.T. Smale. 1992. Similar mechanisms for transcription initiation mediated through a TATA box or an initiator element. J. Biol. Chem. 267: 2823-2830. 


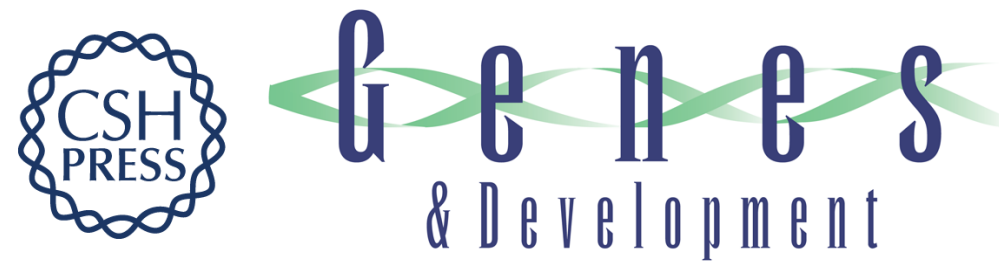

\section{TFIIIC determines RNA polymerase III specificity at the TATA-containing yeast U6 promoter.}

S Roberts, T Colbert and S Hahn

Genes Dev. 1995, 9:

Access the most recent version at doi:10.1101/gad.9.7.832

References This article cites 44 articles, 20 of which can be accessed free at:

http://genesdev.cshlp.org/content/9/7/832.full.html\#ref-list-1

License

Email Alerting

Service right corner of the article or click here.

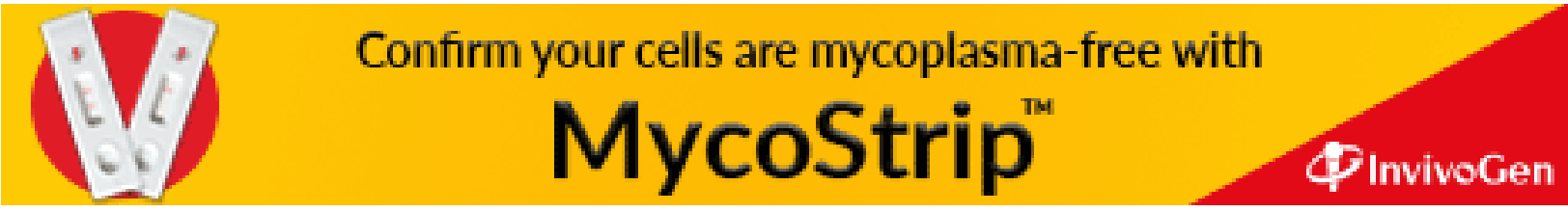

